\title{
Optimal Neumann control for the 1D wave equation: Finite horizon, infinite horizon, boundary tracking terms and the turnpike property
}

\author{
Martin Gugat* Emmanuel Trélat ${ }^{\dagger} \quad$ Enrique Zuazua
}

\begin{abstract}
We consider a vibrating string that is fixed at one end with Neumann control action at the other end. We investigate the optimal control problem of steering this system from given initial data to rest, in time $T$, by minimizing an objective functional that is the convex sum of the $L^{2}$-norm of the control and of a boundary Neumann tracking term.

We provide an explicit solution of this optimal control problem, showing that if the weight of the tracking term is positive, then the optimal control action is concentrated at the beginning and at the end of the time interval, and in-between it decays exponentially. We show that the optimal control can actually be written in that case as the sum of an exponentially decaying term and of an exponentially increasing term. This implies that, if the time $T$ is large, then the optimal trajectory approximately consists of three arcs, where the first and the third shorttime arcs are transient arcs, and in the middle arc the optimal control and the corresponding state are exponentially close to 0 . This is an example of a turnpike phenomenon for a problem of optimal boundary control. If $T=+\infty$ (infinite time horizon problem), then only the exponentially decaying component of the control remains, and the norms of the optimal control action and of the optimal state decay exponentially in time. In contrast to this situation, if the weight of the tracking term is zero and only the control cost is minimized, then the optimal control is distributed uniformly along the whole interval $[0, T]$ and coincides with the control given by the Hilbert Uniqueness Method.

In addition, we establish a similarity theorem stating that, for every $T>0$, there exists an appropriate weight $\lambda<1$ for which the optimal solutions of the corresponding finite horizon optimal control problem and of the infinite horizon optimal control problem coincide along the first part of the time interval $[0,2]$. We also discuss the turnpike phenomenon from the perspective of a general framework with a strongly continuous semi-group.
\end{abstract}

Keywords: Vibrating string, Neumann boundary control, turnpike phenomenon, exponential stability, energy decay, exact control, infinite horizon optimal control, similarity theorem, receding horizon.

\section{Introduction}

The turnpike property states that, loosely speaking, if the objective function of an optimal control problem penalizes both control cost and the difference of the optimal trajectory to a given desired

\footnotetext{
*FAU, Friedrich-Alexander-University Erlangen-Nürnberg, Department Mathematik, Cauerstr. 11, 91058 Erlangen, Germany (martin.gugat@fau.de)

†Sorbonne Universités, UPMC Univ Paris 06, CNRS UMR 7598, Laboratoire Jacques-Louis Lions, Institut Universitaire de France, F-75005, Paris, France (emmanuel.trelat@upmc.fr).

${ }^{\ddagger}$ Departamento de Matemáticas, Universidad Autónoma de Madrid, Cantoblanco 28049 Madrid, Spain (enrique.zuazua@uam.es).
}

(C) 2016. This manuscript version is made available under the Elsevier user license http://www.elsevier.com/open-access/userlicense/1.0/ 
stationary state, then the optimal controls will steer the system quickly to the desired stationary state and then the system will remain on this path most of the time.

Turnpike theory has originally been discussed in econometry (see [4), and then has been developed and generalized in control theory in more general contexts. For the turnpike phenomenon for infinite dimensional systems we refer the reader to [1] and to the many references therein. Recently the turnpike property has been discussed for the optimal control of linear systems governed by partial differential equations with distributed control in [20]. The turnpike property in finite dimension has been studied for example in [12, 18, 21, 17, 23]. Zaslavski has contributed to the study of the turnpike phenomenon for example in [25, 26, 27, 28, In [3] dissipative discrete time optimal control problems have been considered. In [20, Section 4], optimal control problems with the wave equation are considered on a given finite time interval $[0, T]$. The control is distributed in the interior of the domain and no conditions for the terminal state at the time $T$ are prescribed.

In this paper we consider problems of optimal boundary control of the wave equation. We consider both problems with infinite time horizon and problems with finite time horizon. For the finite time problems, we prescribe exact terminal conditions. If the objective function only penalizes the control cost, for the $1 D$ case in [7] it has been shown that the optimal controls are periodic, so in particular they do not have the turnpike structure. This illustrates that the turnpike property depends on the choice of the objective function. At least in the absence of constraints it has to couple the control cost with the penalization of the distance to the desired state.

We consider a system governed by the one-dimensional wave equation on a finite space interval, with a homogeneous Dirichlet boundary condition at one side, and a Neumann boundary control action at the other:

$$
\begin{aligned}
& \partial_{t t} y(t, x)=\partial_{x x} y(t, x), \quad(t, x) \in \mathbb{R} \times(0,1), \\
& y(t, 0)=0, \partial_{x} y(t, 1)=u(t), \quad t \in[0, T],
\end{aligned}
$$

where the control $u$ belongs to the class of square-integrable functions.

Let $y_{0} \in H^{1}(0,1)$ be such that $y_{0}(0)=0$, and let $y_{1} \in L^{2}(0,1)$ be arbitrary. For every $u \in L^{2}(0,+\infty)$, there exists a unique solution $y \in C^{0}\left(0,+\infty ; H^{1}(0,1)\right) \cap C^{1}\left(0,+\infty ; L^{2}(0,1)\right)$ of 1 ) such that $y(0, \cdot)=y_{0}(\cdot)$ and $\partial_{t} y(0, \cdot)=y_{1}(\cdot)$.

As is well known, the system is exactly controllable if and only if $T \geqslant 2$ (see for example [9]). In this paper, given any $\lambda \in[0,1]$ and any $T \in[0,+\infty]$, we consider the optimal control problem $(\mathbf{O C P})_{\lambda}^{\mathbf{T}}$ of finding a control $u \in L^{2}(0, T)$ minimizing the objective functional

$$
J_{\lambda}^{T}(u)=\int_{0}^{T}\left((1-\lambda)\left(\partial_{x} y(t, 0)\right)^{2}+\lambda u(t)^{2}\right) d t,
$$

such that the corresponding solution of (1), with $y(0, \cdot)=y_{0}(\cdot)$ and $\partial_{t} y(0, \cdot)=y_{1}(\cdot)$, satisfies $y(T, \cdot)=\partial_{t} y(T, \cdot)=0$ at the final time (exact null controllability problem). If $T=+\infty$, then one can drop the final constraint requirement, which, by the way, happens to be automatically satisfied in the sense of a limit, since we are then within the framework of the well known Riccati theory.

For $\lambda=1$, this classical optimal control problem (minimization of the $L^{2}$-norm of the control) has been studied for example in [14, 16, 22] and it turns out that the optimal control is periodic (see [7), with a period equal to 4 , that is, twice the time needed by a wave starting at the boundary point where the control acts to return to that point. Note that, in this case, the optimal control is as well given by the Hilbert Uniqueness Method (see [16]).

If $\lambda<1$, then the objective functional involves a nontrivial boundary tracking term. This tracking term may be considered as a boundary observation of the space derivative of the state at the uncontrolled end of the string. As we are going to prove, in that case, the optimal control action is then essentially concentrated at the beginning and at the end of the time interval $[0, T]$. 
More precisely, the optimal control can be written as the sum of an exponentially decaying term and of an exponentially increasing term.

As a consequence, if $T$ is large then the optimal control, solution of $(\mathbf{O C P})_{\lambda}^{\mathbf{T}}$, approximately consists of three pieces: the first and the third pieces are in short-time, and are transient arcs; the middle arc is in long time, and is exponentially close to 0 . This is a turnpike phenomenon, meaning that the optimal trajectory, starting from given initial data, very quickly approximately reaches the steady-state $(0,0)$ (within exponentially short time, say $\varepsilon \ll T$ ), then remains exponentially close to that steady-state within long time (say, over the time interval $[\varepsilon, T-\varepsilon]$ ), and, in the last short-time part $[T-\varepsilon, T]$, leaves this neighborhood in order to quickly reach its target.

In this approximate picture, if $T=+\infty$ (infinite horizon), then the last transient arc does not exist since the infinite-horizon target is the steady-state $(0,0)$. In that case, the norm of the optimal control decays exponentially in time, and the same is true for the optimal state. Indeed, smallness of the observation term for a sufficiently long time interval with zero control implies proportional smallness of the state (this follows from an observability inequality, see [29, (3.3)]).

Another possible picture illustrating the turnpike behavior is the following. For $T$ large, the optimal trajectory of $(\mathbf{O C P})_{\lambda}^{\mathbf{T}}$ approximately consists of three arcs: the first arc is the solution of $(\mathbf{O C P})_{\lambda}^{\infty}$ (infinite horizon problem), forward in time, and converges exponentially to 0 . The second arc, occupying the main (middle) part of the time interval, is the steady-state 0 . The third arc is the solution of $(\mathbf{O C P})_{\lambda}^{\infty}$, but backward in time. Note that the optimal control problem $(\mathbf{O C P})_{\lambda}^{\infty}$ fits into the well known Linear Quadratic Riccati theory.

In all cases, we will provide completely explicit formulas for the optimal controls, which explain and imply the turnpike behavior observed for $\lambda<1$. This is in contrast with the case $\lambda=1$ for which the control action is distributed uniformly along the time interval $[0, T]$. In addition, we will also establish a similarity theorem showing that, for every $T \geqslant 2$ there exists an appropriate weight $\lambda(T)<1$ for which the optimal solutions of $(\mathbf{O C P})_{1}^{\mathbf{T}}$ and of $(\mathbf{O C P})_{\lambda(\mathbf{T})}^{\infty}$ coincide along the first part of the time interval $[0,2]$. This result justifies a receding horizon control strategy, where the first part of a finite horizon optimal control is used and then the procedure is updated in order to control the system over an infinite time horizon.

This paper has the following structure. In Section 2 we present the main results about the Neumann-boundary control of the vibrating string. In 2.4 some numerical illustrations are presented. Section 3 contains the proofs for the results from Section 2 , In Section 4 , we consider the turnpike phenomenon from a more general viewpoint for an optimal control problem with an evolution that is governed by a strongly continuous semigroup.

\section{The main results}

Now we come to our results about optimal control problems for a system governed by (1). Let $y_{0} \in H^{1}(0,1)$ be such that $y_{0}(0)=0$, and let $y_{1} \in L^{2}(0,1)$ be arbitrary.

\subsection{Explicit optimal controls}

We have the following result, giving the explicit solution of $(\mathbf{O C P})_{\lambda}^{\mathbf{T}}$, for any $\lambda \in[0,1]$ and any $T \in[2,+\infty]$. Note that, when $T=+\infty$, we have to assume that $\lambda<1$ to ensure well-posedness.

Theorem 1. For every $T \in[2,+\infty]$ and every $\lambda \in[0,1]$, the problem $(\mathbf{O C P})_{\lambda}^{\mathbf{T}}$ has a unique optimal control solution denoted by $u_{\lambda}^{T}$. 
1. We assume that $T$ is finite. Define

$$
K(T)=\max \{n \in\{1,2,3, \ldots\}: 2 n \leqslant T\}
$$

and

$$
\Delta(T)=T-2 K(T)
$$

For $t \in[0,2)$, let

$$
d(t)=\left\{\begin{aligned}
K(T)+1, & t \in[0, \Delta(T)), \\
K(T), & t \in[\Delta(T), 2) .
\end{aligned}\right.
$$

If $\lambda<1$, then the optimal control solution of $(\mathbf{O C P})_{\lambda}^{\mathbf{T}}$ is the sum of an exponentially decaying term and of an exponentially increasing one. More precisely, defining the real number $z_{\lambda} \in$ $(-1,0)$ by

$$
z_{\lambda}=\frac{-\lambda}{2-\lambda+2 \sqrt{1-\lambda}},
$$

we have

$$
u_{\lambda}^{T}(t+2 k)=z_{\lambda}^{k} f_{+}(t)+\frac{1}{z_{\lambda}^{k}} f_{-}(t),
$$

for every $t \in(0,2)$ and every $k \in \mathbb{N}$ such that $t+2 k \leqslant T$, where

$$
f_{+}(t)=\frac{1+z_{\lambda}}{1-z_{\lambda}^{2 d(t)}} F(t-1), \quad f_{-}(t)=\frac{1+\frac{1}{z_{\lambda}}}{1-\frac{1}{z_{\lambda}^{2 d(t)}}} F(t-1),
$$

with $F \in L^{2}(-1,1)$ defined by

$$
F(t)= \begin{cases}\frac{1}{2}\left(y_{0}^{\prime}(-t)-y_{1}(-t)\right), & t \in(-1,0), \\ \frac{1}{2}\left(y_{0}^{\prime}(t)+y_{1}(t)\right), & t \in[0,1) .\end{cases}
$$

2. We assume that $T=+\infty$ and that $\lambda<1$.

If $\lambda=0$, then the optimal control $u_{0}^{\infty}$, solution of $(\mathbf{O C P})_{\mathbf{0}}^{\infty}$, coincides along the time interval $[0,2]$ with the optimal control $u_{1}^{2}$, solution of $(\mathbf{O C P})_{\mathbf{1}}^{\mathbf{2}}$.

If $0<\lambda<1$, the optimal control $u_{\lambda}^{\infty}$, solution of $(\mathbf{O C P})_{\lambda}^{\infty}$, is given along the time interval $[0,2]$ by

$$
u_{\lambda}^{\infty}(t)= \begin{cases}\frac{1+z_{\lambda}}{2}\left(y_{0}^{\prime}(1-t)-y_{1}(1-t)\right), & t \in(0,1), \\ \frac{1+z_{\lambda}}{2}\left(y_{0}^{\prime}(t-1)+y_{1}(t-1)\right), & t \in(1,2),\end{cases}
$$

and moreover, we have

$$
u_{\lambda}^{\infty}(t+2 k)=z_{\lambda}^{k} u_{\lambda}^{\infty}(t),
$$

for every $t \in(0,2)$ and every $k \in\{1,2,3, \ldots\}$.

The corresponding optimal state $y_{\lambda}^{\infty}$ decays exponentially, in the sense that there exists $C_{0}>0$ such that

$$
\begin{aligned}
\int_{0}^{1}\left(\left(\partial_{x} y_{\lambda}^{\infty}(t+2 k, x)\right)^{2}+\left(\partial_{t} y_{\lambda}^{\infty}(t+2 k, x)\right)^{2}\right) & d x \\
& \leqslant C_{0}\left|z_{\lambda}\right|^{2 k} \int_{0}^{1}\left(y_{0}^{\prime}(x)^{2}+y_{1}(x)^{2}\right) d x
\end{aligned}
$$

for every $t \in(0,2)$ and every $k \in \mathbb{N}^{*}$. 
Theorem 1 is proved in Section 3.2 .

For $\lambda=1$, that is, when there is no tracking term in the objective functional, the explicit solution of $(\mathbf{O C P})_{1}^{\mathbf{T}}$ given above has already been computed in [7, Theorem 2.1], see also [8] (which does not provide any results on the turnpike phenomenon). In this case, the problem consists of minimizing the $L^{2}$-norm of the (Neumann) control. The optimal control $u_{1}^{T}$, whose explicit formula is given above, can also be characterized as well with the famous Hilbert Uniqueness Method (see [16]) and is then often referred to as the HUM control.

Here, there is no dissipation induced by the objective functional (no tracking term), the optimal control is periodic, and is uniformly distributed over the time interval $[0, T]$, in the sense that there is no energy decay.

In contrast, if $\lambda<1$, the control is the sum of two terms, one of which is exponentially decreasing, and the other being exponentially increasing. For $T$ large enough, this implies the turnpike phenomenon, stated in details in Section 2.2 .

In fact in Theorem 1 the turnpike is the zero state: Starting from the initial state, the system approaches this turnpike exponentially fast. In order to reach the zero state exactly at the final time $T$, that is to satisfy the terminal conditions, additional control effort is necessary close to the final time. The exponential decay depends on the size of $z_{\lambda}$ (that is on the decay of $\left.\left(z_{\lambda}^{k}\right)_{k}\right)$, which depends in turn continuously on $\lambda$. In this sense, the decay depends continuously on $\lambda$. Since $\lim _{\lambda \rightarrow 1} z_{\lambda}=-1$, as $\lambda$ approaches 1 the exponential decay becomes slower until it vanishes for $\lambda=1$.

Remark 1. For $\lambda=0$, the solution of $(\mathbf{O C P})_{\mathbf{0}}^{\infty}$ coincides with the solution of the problem of optimal feedback control studied in [11].

Remark 2. The estimate (9) is clearly equivalent to

$$
\int_{0}^{1}\left(\left(\partial_{x} y_{\lambda}^{\infty}(t, x)\right)^{2}+\left(\partial_{t} y_{\lambda}^{\infty}(t, x)\right)^{2}\right) d x \leqslant C_{1} e^{-\mu t} \int_{0}^{1}\left(y_{0}^{\prime}(x)^{2}+y_{1}(x)^{2}\right) d x
$$

for every time $t \geqslant 0$, for some positive constants $C_{1}$ and $\mu$ not depending on the initial state (see also [10, Lemma 2]).

Remark 3. It is well known that the solution of the infinite horizon problem $(\mathbf{O C P})_{\lambda}^{\infty}$ can also be expressed in feedback form (Linear Quadratic Riccati theory, see for example [6]). Hence, in the case $T=+\infty$, our result is certainly not new but can rather be seen as a reinterpretation of the well known results coming from the Riccati theory. More precisely, the velocity feedback

$$
\partial_{x} y(t, 1)=\frac{z_{\lambda}+1}{z_{\lambda}-1} \partial_{t} y(t, 1)
$$

generates the same state as the one generated by the optimal control $u_{\lambda}^{\infty}$. We stress however that our point of view is different and that we provide explicit formulas that express the optimal control in terms of the initial state.

Remark 4. In the above results, we considered only the null steady-state, but we can easily replace it with any other steady-state that is compatible with the boundary condition $y(t, 0)=0$ as follows. 
Any such steady-state of (1) is given by $\bar{y}(x)=\sigma x$, for some $\sigma \in \mathbb{R}$. The corresponding optimal boundary control problem for the finite time horizon $T>0$ is

$$
\left\{\begin{array}{l}
\min \int_{0}^{T}\left((1-\lambda)\left(\partial_{x} y(t, 0)-\sigma\right)^{2}+\lambda u(t)\right) d t \\
\text { subject to } \\
y(0, x)=\sigma x+y_{0}(x), \partial_{t} y(T, x)=y_{1}(x), \quad x \in(0,1), \\
y(t, 0)=0, \partial_{x} y(t, 1)=\sigma+u(t), \quad t \in(0, T), \\
\partial_{t t} y(t, x)=\partial_{x x} y(t, x), \quad(t, x) \in(0, T) \times(0,1), \\
y(T, x)=\sigma x, \partial_{t} y(T, x)=0, \quad x \in(0,1) .
\end{array}\right.
$$

The corresponding optimal control is the same as in Theorem 1 . The transformation for the infinite horizon case is similar.

In the finite horizon case in our system the time-direction can be reversed, so if the control is transformed accordingly, it can be used to control the system from a stationary state $(\sigma x, 0)$ to $\left(y_{0}, y_{1}\right)$ in an optimal way.

Remark 5. In Theorem 1 we present the optimal controls as functions of the initial states. In the control of engineering systems, the initial state are usually not known exactly. However, often the initial state can be estimated using observations from the past. Let us illustrate this for the finite time horizon case. Our results in Theorem 1 imply that for every $T>0$ the optimal control $u \in L^{2}(0, T)$ is given as the image of a linear map of $y_{0}^{\prime}$ and $y_{1} \in L^{2}(0,1)$ that is continuous in the sense that

$$
\int_{0}^{T} u(\tau)^{2} d \tau \leqslant C_{r}^{2} \int_{0}^{1}\left(y_{0}^{\prime}(x)\right)^{2}+y_{1}(x)^{2} d x
$$

with a constant $C_{r}$ that does not depend on $y_{0}^{\prime}$ and $y_{1}$. In this sense the control is stable with respect to the $L^{2}$-norm. The application of a control that is optimal for an estimated initial state $\left(y_{0}^{\text {est }}, y_{1}^{\text {est }}\right)$ steers the system to a state with an $L^{2}-$ norm of the order of the $L^{2}$-error of the estimation of the initial state. The reason is the well-known fact that with zero control, our system conserves the energy $\int_{0}^{1}\left(\partial_{x} y(t, x)\right)^{2}+\left(\partial_{t} y(t, x)\right)^{2} d x$ (see for example [8], Theorem 2.4). So with the optimal control for $\left(y_{0}^{\text {est }}, y_{1}^{\text {est }}\right)$ applied to the system with the initial state $\left(y_{0}, y_{1}\right)$, at the time $T$ the state of the system will have the same energy as the error $\left(y_{0}-y_{0}^{e s t}, y_{1}-y_{1}^{\text {est }}\right)$. In order to steer this state to rest, the feedback control from Remark 3 can be used.

\subsection{Consequence: the turnpike behavior}

From Theorem 1 and from the previous discussions, we infer the following consequence on the qualitative behavior of the optimal solution.

Corollary 1. For every $\lambda \in[0,1)$, then there exist $C_{1}>0$ and $\mu>0$ such that, for every $T \geqslant 2$, for all initial conditions $\left(y_{0}, y_{1}\right) \in H^{1}(0,1) \times L^{2}(0,1)$ with $y_{0}(0)=0$, the optimal solution of $(\mathbf{O C P})_{\lambda}^{\mathbf{T}}$ satisfies the estimate

$$
\int_{0}^{1}\left(\left(\partial_{x} y_{\lambda}^{T}(t, x)\right)^{2}+\left(\partial_{t} y_{\lambda}^{T}(t, x)\right)^{2}\right) d x \leqslant C_{1} e^{-\mu t(T-t)} \int_{0}^{1}\left(y_{0}^{\prime}(x)^{2}+y_{1}(x)^{2}\right) d x
$$

for every $t \in[0, T]$.

In the estimate (11), what is important to see is that the term $e^{-\mu t(T-t)}$ is equal to 1 at times $t=0$ and $t=T$, but it is exponentially small in the middle of the interval. It becomes even smaller and smaller when $T$ is taken larger. This estimate implies the turnpike behavior described previously: short-time arcs at the beginning and at the end of the interval are devoted to satisfy the terminal constraints, and in-between, the trajectory remains essentially close to rest. 


\subsection{Similarity result}

We next state the following similarity result: for any final time $T \geqslant 2$ that is a positive even integer, there exists a weight $\lambda$ such that the optimal solutions of $(\mathbf{O C P})_{1}^{\infty}$ and $(\mathbf{O C P})_{\lambda}^{\infty}$ coincide along the subinterval $[0,2]$ of $[0, T]$. More generally, for any real number $T \geqslant 2$, there exists a weight $\lambda$ such that the optimal solutions of $(\mathbf{O C P})_{1}^{\infty}$ and $(\mathbf{O C P})_{\lambda}^{\infty}$ coincide along the subinterval $[0, \Delta(T)]$ of $[0, T]$.

Theorem 2. Given any $T \in\{2,4,6, \ldots\}$ we choose $\lambda>0$ such that

$$
z_{\lambda}=\frac{2}{T}-1
$$

Then we have

$$
u_{1}^{T}(t)=u_{\lambda}^{\infty}(t), \quad \text { for all } t \in(0,2),
$$

and

$$
\left\|u_{1}^{T}(\cdot)-u_{\lambda}^{\infty}(\cdot)\right\|_{L^{2}(2 k, 2 k+2)} \leqslant\left(1-\left|z_{\lambda}\right|^{k}\right) \frac{2}{T}\left(\left\|y_{0}^{\prime}\right\|_{L^{2}(0,1)}+\left\|y_{1}\right\|_{L^{2}(0,1)}\right) .
$$

for every $k \in\{0,1, \ldots,(T-2) / 2\}$.

Given any $T>2, T \notin\{2,4,6, \ldots\}$ we choose $\lambda>0$ such that

$$
z_{\lambda}=\frac{1}{K(T)+1}-1
$$

Then we have

$$
u_{1}^{T}(t)=u_{\lambda}^{\infty}(t), \quad \text { for all } t \in(0, \Delta(T))
$$

Remark 6. Note that for the case that $T$ is an even integer the length of the intervals where the optimal controls coincide is maximal.

The proof of Theorem 2 is done in Section 3.3 .

\subsection{Numerical illustration}

We set $y_{0}(x)=4 \sin (\pi x / 2)$ and $y_{1}(x)=0$, for every $x \in[0,1]$. From Theorem 1 , if $0<\lambda<1$ then the optimal control solution of $(\mathbf{O C P})_{\lambda}^{\infty}$ is given by

$$
u_{\lambda}^{\infty}(t+2 k)=\left|z_{\lambda}\right|^{k}\left(1+z_{\lambda}\right) \pi \sin \left(\frac{\pi}{2}(t+2 k)\right),
$$

for $t \in(0,2)$ and $k \in \mathbb{N}$.

The graph of $\partial_{x} y_{\lambda}^{\infty}(t, x)$ is provided on Figure 1 , on the time interval $[0,20]$ with $\lambda=24 / 25$ on Figure 1(a) and $\lambda=99 / 100$ on Figure 1(b). The control $u_{\lambda}^{\infty}(t)=\partial_{x} y_{\lambda}^{\infty}(t, 1)$ is the boundary trace at the back.

These figures illustrate that the norm of the optimal state decays faster if $\lambda$ is smaller, as expected. However, smaller values of $\lambda$ cause larger oscillations. Note that $z_{\lambda}=-2 / 3$ if $\lambda=$ $24 / 25$, and $z_{\lambda}=-9 / 11$ if $\lambda=99 / 100$. Moreover, as pointed out in [7, if $T \in 2 \mathbb{N}^{*}$ then $u_{1}^{T}(t+$

$2 k)=\frac{2}{T} \pi \sin \left(\frac{\pi}{2}(t+2 k)\right.$ ), for all $t$ and $k$ such that $t+2 k \in[0, T]$ (see also [7, Figure 4] for the corresponding optimal state, with $T=10$, up to the factor $2 \pi$ ). 


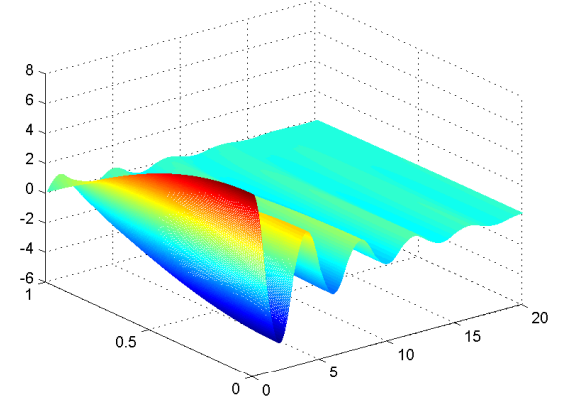

(a) $\lambda=24 / 25$ and $T=20$.

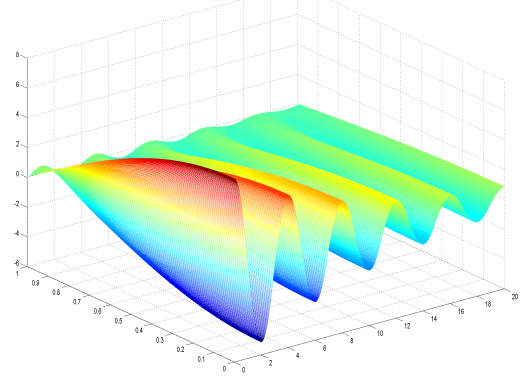

(b) $\lambda=99 / 100$ and $T=20$.

Figure 1: Plot of $\partial_{x} y(t, x)$.

\section{Proofs}

\subsection{Well-posedness of the initial-boundary value problem}

Let $y_{0} \in H^{1}(0,1)$ be such that $y_{0}(0)=0$, and let $y_{1} \in L^{2}(0,1)$ be arbitrary. Let $T \geqslant 2$, and let $u \in L^{2}(0, T)$ be fixed. As a preliminary result, we study the well-posedness of the initial boundary value problem (1) for a fixed control $u$, and with the fixed initial data $\left(y_{0}, y_{1}\right)$. The analysis is similar to the one done in [10]. For this purpose we choose $\bar{T} \in\{2,4,6, \ldots\}$ such that $T \leqslant \bar{T}$. Moreover, we extend our control function by zero to a control in $L^{2}(0, \bar{T})$. Then we show that the solution of the initial boundary value problem (1) exists on $(0, \bar{T})$.

We search a solution given as the sum of two traveling waves, i.e.,

$$
y(t, x)=\alpha(x+t)+\beta(x-t),
$$

where the functions $\alpha$ and $\beta$ are to be determined from the initial data and from the boundary data. First of all, to match the initial conditions, we must have

$$
\begin{aligned}
& \alpha(t)=\frac{1}{2}\left(y_{0}(t)+\int_{0}^{t} y_{1}(s) d s\right)+C_{0}, \\
& \beta(t)=\frac{1}{2}\left(y_{0}(t)-\int_{0}^{t} y_{1}(s) d s\right)-C_{0},
\end{aligned}
$$

where $C_{0}$ is a real number. Besides, the boundary condition $y(t, 0)=0$ implies that

$$
\beta(-s)=-\alpha(s)
$$

for almost every $s>0$. The boundary condition at $x=1$ leads to $\alpha^{\prime}(1+t)=u(t)-\beta^{\prime}(1-t)$, and integrating in time, we get

$$
\alpha(t+1)=\beta(1-t)+\int_{0}^{t} u(s) d s+\alpha(1)-\beta(1) .
$$

Using 17 and 18, we have $\alpha(1)-\beta(1)=\int_{0}^{1} y_{1}(s) d s+2 C_{0}$, and therefore, choosing $C_{0}=$ $-\frac{1}{2} \int_{0}^{1} y_{1}(s) d s$, we get $\alpha(1)-\beta(1)=0$ and

$$
\alpha(t+1)=\beta(1-t)+\int_{0}^{t} u(s) d s
$$


Using (19), the values of $\alpha$ for $t \in(0,1)$, given by $(17)$, determine those of $\beta$ for $t \in(-1,0)$. The values of $\beta$ for $t \in(0,1)$ are given by $(18)$. Now, knowing $\beta$ on the interval $(-1,1)$, we deduce from (20) the values of $\alpha$ on the interval $(1,3)$.

Using $\sqrt{19}$, we get $\alpha(t+1)=-\alpha(t-1)+\int_{0}^{t} u(s) d s$, for $t \geqslant 1$, or equivalently,

$$
\alpha(t+2)=-\alpha(t)+\int_{0}^{t+1} u(s) d s
$$

for $t \geqslant 0$.

Using (21) enables us to determine $\alpha$ iteratively: starting with $\alpha$ on the interval $(1,3)$, the values of $u(t)$ yield those of $\alpha$ on $(3,5)$, and then using (21), we determine $\alpha$ on $(7,9)$, etc.

In order to express everything in terms of $\alpha$ only (without using $\beta$ ), we extend the domain of $\alpha$ so that it contains $(-1,0)$. We get the values of $\alpha$ on $(-1,0)$ by using $\sqrt{19} p$ for $s \in(0,1)$, which yields $\alpha(t)=-\beta(-t)$ for $t \in(-1,0)$ with the values of $\beta$ on $(0,1)$ given by (18). Then, using (20), we get $\alpha(t+2)=-\alpha(t)+\int_{0}^{t+1} u(s) d s$ for $t \in(-1,0)$. We have the following lemma.

Lemma 1. Let $y_{0} \in H^{1}(0,1)$ be such that $y_{0}(0)=0$, and let $y_{1} \in L^{2}(0,1)$ be arbitrary. We set

$$
C_{0}=-\frac{1}{2} \int_{0}^{1} y_{1}(s) d s
$$

and we define $\alpha \in L^{2}(-1,1)$ by

$$
\alpha(t)= \begin{cases}\frac{1}{2}\left(-y_{0}(-t)+\int_{0}^{-t} y_{1}(s) d s\right)+C_{0} & \text { if } \quad t \in(-1,0), \\ \frac{1}{2}\left(y_{0}(t)+\int_{0}^{t} y_{1}(s) d s\right)+C_{0} & \text { if } \quad t \in[0,1) .\end{cases}
$$

Let $\bar{T} \in\{2,4,6, \ldots\}$, and let $u \in L^{2}(0, \bar{T})$ be fixed.

The function $\alpha$, defined by iteration according to

$$
\alpha(t+2 k)=-\alpha(t+2(k-1))+\int_{0}^{t+2 k-1} u(s) d s,
$$

for every $t \in(-1,1)$ and every $k \in \mathbb{N}$ such that $t<\bar{T}+1-2 k$, is well defined on the interval $(-1, \bar{T}+1)$, and belongs to $H^{1}(-1, \bar{T}+1)$.

Proof: From the construction, it is clear that $\left.\alpha\right|_{(k-1, k)} \in H^{1}(k, k+1)$, for every $k \in \mathbb{N}$. To prove that $\alpha \in H^{1}(-1, \bar{T}+1)$, it suffices to prove that $\alpha$ is continuous. Since $\alpha\left(0^{+}\right)=\alpha\left(0^{-}\right)=C_{0}$, $\alpha$ is continuous at $t=0$. Using $(23), \alpha$ is continuous as well on $(-1,1)$.

At $t=1$, using 22 we get $\alpha\left(1^{+}\right)=\alpha\left(1^{-}\right)=\frac{1}{2} y_{0}(1)$, and hence $\alpha$ is continuous at $t=1$. Then, at this step, we have obtained that $\alpha$ is continuous on $(-1,3)$.

We then proceed by induction. Let $k \in \mathbb{N}^{*}$. We assume that $\alpha$ is continuous on the interval $(-1,1+2 k)$. Then $\alpha\left((-1+2 k)^{-}\right)=\alpha\left((-1+2 k)^{+}\right)$. Using (24), we have

$$
\begin{aligned}
& \alpha((1+2 k)-)=-\alpha\left((1+2(k-1))^{-}\right)+\int_{0}^{2 k} u(s) d s=-\alpha\left((-1+2 k)^{-}\right)+\int_{0}^{2 k} u(s) d s \\
&=-\alpha\left((-1+2 k)^{+}\right)+\int_{0}^{2 k} u(s) d s=\alpha\left((-1+2(k+1))^{+}\right)=\alpha\left((1+2 k)^{+}\right) .
\end{aligned}
$$

Since $\alpha$ is defined by $(23)$, we infer that $\alpha$ is continuous on $(-1,1+2(k+1))$ for $k+1 \leqslant \bar{T} / 2$. Lemma 1 is proved. 
Using Lemma 1, we are now in a position to compute the solution of the initial boundary value problem under consideration in this subsection.

Proposition 1. Let $y_{0} \in H^{1}(0,1)$ be such that $y_{0}(0)=0$, and let $y_{1} \in L^{2}(0,1)$ be arbitrary. Let $\bar{T} \in\{2,4,6, \ldots\}$, and let $u \in L^{2}(0, \bar{T})$ be fixed. We consider the function $\alpha$ defined in Lemma 1 by 23). Then the solution of (1), associated with the control $u$ and with the initial data $\left(y_{0}, y_{1}\right)$, is given by

$$
y(t, x)=\alpha(t+x)-\alpha(t-x),
$$

for all $(t, x) \in(0, \bar{T}) \times(0,1)$.

Proof: The construction of $\alpha$ implies that $y$, defined by (25), is a solution of the initial boundary value problem under consideration. We conclude by Cauchy uniqueness.

\subsection{Proof of Theorem 1}

In this section we prove Theorem 1 using the representation of the state presented in Section 3.1 .

\subsubsection{The Case $T<\infty$}

Define $\bar{T}=2 K(T)+2$. The control $u_{\lambda}^{T}$ defined in the theorem is in $L^{2}(0, T)$. Let $y_{\lambda}^{T}$ be the state generated by $u_{\lambda}^{T}$.

Let us first prove that $y_{\lambda}^{T}$ satisfies the terminal constraints

$$
y_{\lambda}^{T}(T, \cdot)=0, \quad \partial_{t} y_{\lambda}^{T}(T, \cdot)=0 .
$$

It suffices to prove that $\alpha_{\lambda}^{\prime}(z)=0$, for $z \in(T-1, T+1)$. From 25 , we have $y_{\lambda}^{T}(t, x)=$ $\alpha_{\lambda}(t+x)-\alpha_{\lambda}(t-x)$, with $\alpha_{\lambda}$ defined by (23). The definition (6) implies that $F(t)=\alpha_{\lambda}^{\prime}(t)$. Hence, we have

$$
\begin{aligned}
\alpha_{\lambda}^{\prime}(t-1) & =\frac{1-z_{\lambda}^{2 d(t)}}{1-z_{\lambda}^{2 d(t)}} F(t-1) \\
& =\left(\frac{1}{1-z_{\lambda}^{2 d(t)}}+\frac{z_{\lambda}^{2 d(t)}}{z_{\lambda}^{2 d(t)}-1}\right) F(t-1) \\
& =\left(\frac{1}{1-z_{\lambda}^{2 d(t)}}+\frac{1}{1-z_{\lambda}^{-2 d(t)}}\right) F(t-1) \\
& =\frac{1}{1+z_{\lambda}} f_{+}(t)+\frac{1}{1+\frac{1}{z_{\lambda}}} f_{-}(t),
\end{aligned}
$$

where the last equality follows from (5).

By (24) we have

$$
\alpha_{\lambda}^{\prime}(t+1)=-\alpha_{\lambda}^{\prime}(t-1)+u_{\lambda}^{T}(t),
$$

for $t \in(0,2)$. Using (4), this yields $\alpha_{\lambda}^{\prime}(t+1)=-\alpha_{\lambda}^{\prime}(t-1)+f_{+}(t)+f_{-}(t)$, for $t \in(0,2)$, and then, using (5),

$$
\alpha_{\lambda}^{\prime}(t+1)=\frac{z_{\lambda}}{1+z_{\lambda}} f_{+}(t)+\frac{\frac{1}{z_{\lambda}}}{1+\frac{1}{z_{\lambda}}} f_{-}(t)
$$


By induction, thanks to (27) and (4), this implies that

$$
\alpha_{\lambda}^{\prime}(t-1+2 k)=\frac{z_{\lambda}^{k}}{1+z_{\lambda}} f_{+}(t)+\frac{\frac{1}{z_{\lambda}^{k}}}{1+\frac{1}{z_{\lambda}}} f_{-}(t),
$$

for every $t \in(0,2)$ and every $k \in \mathbb{N}$ such that $t+2 k \leqslant T+2$. Taking $k=K(T)$, we get

$$
\alpha_{\lambda}^{\prime}(t-1+2 K(T))=\frac{z_{\lambda}^{K(T)}}{1+z_{\lambda}} f_{+}(t)+\frac{\frac{1}{z_{\lambda}^{K(T)}}}{1+\frac{1}{z_{\lambda}}} f_{-}(t) .
$$

Moreover, since $T=2 K(T)+\Delta(T)$, for $t \in[0, \Delta(T))$ we can take $k=K(T)+1$ and get

$$
\alpha_{\lambda}^{\prime}(t+1+2 K(T))=\frac{z_{\lambda}^{K(T)+1}}{1+z_{\lambda}} f_{+}(t)+\frac{\frac{1}{z_{\lambda}^{K(T)+1}}}{1+\frac{1}{z_{\lambda}}} f_{-}(t) .
$$

Considering the cases $t \in(0, \Delta(T))$ and $t \in[\Delta(T), 2)$ separately, using (5), we infer that

$$
\alpha_{\lambda}^{\prime}(t-1+T)=\left(\frac{z_{\lambda}^{d(t)}}{1-z_{\lambda}^{2 d(t)}}+\frac{\frac{1}{z_{\lambda}^{d(t)}}}{1-\frac{1}{z_{\lambda}^{2 d(t)}}}\right) F(t-1)=0,
$$

and hence the state $y_{\lambda}^{T}$ satisfies the terminal conditions 26 .

For a control of the form $u=u_{\lambda}^{T}+h$, the generated state is $y=y_{\lambda}^{T}+y_{h}$, where $y_{h}$ is the state generated by the perturbation control $h$, with the boundary conditions $y_{h}(t, 0)=0$, $\partial_{x} y_{h}(t, 1)=h(t)$, and null initial conditions. We only consider variations $h$ for which the terminal conditions $y_{h}(T, \cdot)=0$ and $\partial_{t} y_{h}(T, \cdot)=0$ hold. Using [25), we have

$$
y_{h}(t, x)=\alpha_{h}(t+x)-\alpha_{h}(t-x) .
$$

Due to this representation of $y_{h}$, the initial conditions $y_{h}(0, \cdot)=\partial_{t} y_{h}(0, \cdot)=0$ imply that for $t \in(-1,1)$ we have $\alpha_{h}^{\prime}(t)=0$. Moreover, owing to the terminal constraints, we must have $\alpha_{h}^{\prime}=0$ along $(T-1, T+1)$.

The value of the objective functional of $(\mathbf{O C P})_{\lambda}^{\mathbf{T}}$ is

$$
\begin{aligned}
\int_{0}^{T}((1-\lambda) & \left.\left(\partial_{x} y(t, 0)\right)^{2}+\lambda u(t)^{2}\right) d t \\
=\int_{0}^{T}\left(( 1 - \lambda ) \left(\left(\partial_{x} y_{\lambda}^{T}(t, 0)\right)^{2}+\left(\partial_{x} y_{h}(t, 0)\right)^{2}\right.\right. & \left.+2 \partial_{x} y_{\lambda}^{T}(t, 0) \partial_{x} y_{h}(t, 0)\right) \\
& \left.+\lambda\left(u_{\lambda}^{T}(t)^{2}+h(t)^{2}+2 u_{\lambda}^{T}(t) h(t)\right)\right) d t .
\end{aligned}
$$

We consider the linear part

$$
L_{\lambda}(h)=2 \int_{0}^{T}\left((1-\lambda) \partial_{x} y_{\lambda}^{T}(t, 0) \partial_{x} y_{h}(t, 0)+\lambda u_{\lambda}^{T}(t) h(t)\right) d t .
$$

Since $\partial_{x} y_{\lambda}^{T}(t, 0)=2 \alpha_{\lambda}^{\prime}(t), \partial_{x} y_{h}(t, 0)=2 \alpha_{h}^{\prime}(t)$, and

$$
\begin{aligned}
u_{\lambda}^{T}(t) & =\partial_{x} y_{\lambda}^{T}(t, 1)=\alpha_{\lambda}^{\prime}(t+1)+\alpha_{\lambda}^{\prime}(t-1), \\
h(t) & =\partial_{x} y_{h}(t, 1)=\alpha_{h}^{\prime}(t+1)+\alpha_{h}^{\prime}(t-1),
\end{aligned}
$$


we get

$$
\begin{aligned}
L_{\lambda}(h) & =\int_{0}^{T}\left(8(1-\lambda) \alpha_{\lambda}^{\prime}(t) \alpha_{h}^{\prime}(t)\right. \\
& \left.+2 \lambda\left(\alpha_{\lambda}^{\prime}(t+1)+\alpha_{\lambda}^{\prime}(t-1)\right)\left(\alpha_{h}^{\prime}(t+1)+\alpha_{h}^{\prime}(t-1)\right)\right) d t
\end{aligned}
$$

We extend the domain of the function $\alpha_{h}^{\prime}$ and $\alpha_{\lambda}^{\prime}$ to the interval $(0, \infty)$ by defining $\alpha_{h}^{\prime}(t)=0$ and $\alpha_{\lambda}^{\prime}(t)=0$ for $t \geqslant T+1$. Then we have

$$
\begin{aligned}
L_{\lambda}(h) & =\int_{0}^{\infty}\left(8(1-\lambda) \alpha_{\lambda}^{\prime}(t) \alpha_{h}^{\prime}(t)\right. \\
& \left.+2 \lambda\left(\alpha_{\lambda}^{\prime}(t+1)+\alpha_{\lambda}^{\prime}(t-1)\right)\left(\alpha_{h}^{\prime}(t+1)+\alpha_{h}^{\prime}(t-1)\right)\right) d t \\
& =\sum_{j=0}^{\infty} \int_{0}^{1}\left[8(1-\lambda) \alpha_{\lambda}^{\prime}(t+j) \alpha_{h}^{\prime}(t+j)\right. \\
& \left.+2 \lambda\left(\alpha_{\lambda}^{\prime}(t+1+j)+\alpha_{\lambda}^{\prime}(t-1+j)\right)\left(\alpha_{h}^{\prime}(t+1+j)+\alpha_{h}^{\prime}(t-1+j)\right)\right] d t \\
& =8(1-\lambda) \sum_{j=1}^{\infty} \int_{0}^{1} \alpha_{h}^{\prime}(t+j) \alpha_{\lambda}^{\prime}(t+j) d t \\
& +2 \lambda \sum_{j=1}^{\infty} \int_{0}^{1} \alpha_{h}^{\prime}(t+j)\left(\alpha_{\lambda}^{\prime}(t+2+j)+\alpha_{\lambda}^{\prime}(t+j)\right) d t \\
& +2 \lambda \sum_{j=1}^{\infty} \int_{0}^{1} \alpha_{h}^{\prime}(t+j)\left(\alpha_{\lambda}^{\prime}(t+j)+\alpha_{\lambda}^{\prime}(t-2+j)\right) d t .
\end{aligned}
$$

Since the infinite series in the last equation are in fact finite sumes, we can exchange them with the integral and get

$$
\begin{aligned}
L_{\lambda}(h)=2 \int_{0}^{1} & \sum_{j=1}^{\infty} \alpha_{h}^{\prime}(t+j)\left(4(1-\lambda) \alpha_{\lambda}^{\prime}(t+j)\right. \\
& \left.+\lambda\left(\alpha_{\lambda}^{\prime}(t+2+j)+\alpha_{\lambda}^{\prime}(t-2+j)+2 \alpha_{\lambda}^{\prime}(t+j)\right)\right) d t .
\end{aligned}
$$

Defining the characteristic polynomial by

$$
p_{\lambda}(z)=\lambda z^{2}+(4-2 \lambda) z+\lambda
$$

we have $p_{\lambda}\left(z_{\lambda}\right)=0$ and $p_{\lambda}\left(1 / z_{\lambda}\right)=0$. Using $[28)$, we have

$$
\lambda \alpha_{\lambda}^{\prime}(t+2+j)+(4-2 \lambda) \alpha_{\lambda}^{\prime}(t+j)+\lambda \alpha_{\lambda}^{\prime}(t-2+j)=0,
$$

for every $t \in(0,1)$ and every $j \in\{1,2,3, \ldots$,$\} with t+j \leqslant T-1$. This implies that $L_{\lambda}(h)=0$. Now, concerning the value of the objective functional of $(\mathbf{O C P})_{\lambda}^{\mathbf{T}}$, for any $h$ such that $y_{h}(T, \cdot)=0$ and $\partial_{t} y_{h}(T, \cdot)=0$, we infer that

$$
\int_{0}^{T}\left((1-\lambda)\left(\partial_{x} y(t, 0)\right)^{2}+\lambda u(t)^{2}\right) d t \geqslant \int_{0}^{T}\left((1-\lambda)\left(\partial_{x} y_{\lambda}^{T}(t, 0)\right)^{2}+\lambda u_{\lambda}^{T}(t)^{2}\right) d t
$$

with a strict inequality whenever $h \neq 0$. It follows that $u_{\lambda}^{T}$ is the unique optimal solution of $(\mathbf{O C P})_{\lambda}^{\mathbf{T}}$, as soon as $\lambda>0$. If $\lambda=0$, then the result also follows from the representation of $L_{0}(h)$. However, in this case the characteristic polynomial $p_{0}(z)=4 z$ has only one root given by $z_{0}=0$. Theorem 1 is proved for $T<+\infty$. 


\subsubsection{The Case $T=\infty$}

We are going to use the previously established well-posedness results.

Let $y_{\lambda}^{\infty}$ be the state generated by the control $u_{\lambda}^{\infty}$ defined in the theorem. For a control of the form $u=u_{\lambda}^{\infty}+h$, the generated state is $y=y_{\lambda}^{\infty}+y_{h}$, where $y_{h}$ is the state generated by the control $h$, with null initial conditions and with the boundary conditions $y_{h}(t, 0)=0$ and $\partial_{x} y_{h}(t, 1)=h(t)$. The value of the objective functional of $(\mathbf{O C P})_{\lambda}^{\infty}$ is

$$
\begin{aligned}
\int_{0}^{+\infty}\left((1-\lambda)\left(\partial_{x} y(t, 0)\right)^{2}+\lambda u(t)^{2}\right) d t & \\
=\int_{0}^{+\infty}\left(( 1 - \lambda ) \left(\left(\partial_{x} y_{\lambda}^{T}(t, 0)\right)^{2}+\left(\partial_{x} y_{h}(t, 0)\right)^{2}\right.\right. & \left.+2 \partial_{x} y_{\lambda}^{\infty}(t, 0) \partial_{x} y_{h}(t, 0)\right) \\
& \left.+\lambda\left(u_{\lambda}^{\infty}(t)^{2}+h(t)^{2}+2 u_{\lambda}^{\infty}(t) h(t)\right)\right) d t .
\end{aligned}
$$

We consider the linear part

$$
L_{\lambda}(h)=2 \int_{0}^{+\infty}\left((1-\lambda) \partial_{x} y_{\lambda}^{\infty}(t, 0) \partial_{x} y_{h}(t, 0)+\lambda u_{\lambda}^{\infty}(t) h(t)\right) d t .
$$

Using (25), we have $y_{\lambda}^{\infty}(t, x)=\alpha_{\lambda}(t+x)-\alpha_{\lambda}(t-x)$ and $y_{h}(t, x)=\alpha_{h}(t+x)-\alpha_{h}(t-x)$, with $\alpha_{\lambda}$ given by 23$)$, and $\alpha_{h}=0$ on $(-1,1)$. It follows that $\partial_{x} y_{\lambda}^{\infty}(t, 0)=2 \alpha_{\lambda}^{\prime}(t), \partial_{x} y_{h}(t, 0)=2 \alpha_{h}^{\prime}(t)$, and

$$
\begin{aligned}
u_{\lambda}^{\infty}(t) & =\partial_{x} y_{\lambda}^{\infty}(t, 1)=\alpha_{\lambda}^{\prime}(t+1)+\alpha_{\lambda}^{\prime}(t-1), \\
h(t) & =\partial_{x} y_{h}(t, 1)=\alpha_{h}^{\prime}(t+1)+\alpha_{h}^{\prime}(t-1),
\end{aligned}
$$

and therefore,

$$
\begin{aligned}
L_{\lambda}(h)= & \int_{0}^{+\infty}\left(8(1-\lambda) \alpha_{\lambda}^{\prime}(t) \alpha_{h}^{\prime}(t)\right. \\
& \left.\quad+2 \lambda\left(\alpha_{\lambda}^{\prime}(t+1)+\alpha_{\lambda}^{\prime}(t-1)\right)\left(\alpha_{h}^{\prime}(t+1)+\alpha_{h}^{\prime}(t-1)\right)\right) d t \\
= & \sum_{j=0}^{+\infty} \int_{0}^{1}\left(8(1-\lambda) \alpha_{\lambda}^{\prime}(t+j) \alpha_{h}^{\prime}(t+j)\right. \\
& \left.+2 \lambda\left(\alpha_{\lambda}^{\prime}(t+1+j)+\alpha_{\lambda}^{\prime}(t-1+j)\right)\left(\alpha_{h}^{\prime}(t+1+j)+\alpha_{h}^{\prime}(t-1+j)\right)\right) d t \\
= & 2 \int_{0}^{1}\left(4(1-\lambda) \alpha_{h}^{\prime}(t) \alpha_{\lambda}^{\prime}(t)\right. \\
& \left.\quad+\lambda\left(\alpha_{h}^{\prime}(t-1)\left(\alpha_{\lambda}^{\prime}(t+1)+\alpha_{\lambda}^{\prime}(t-1)\right)+\alpha_{h}^{\prime}(t)\left(\alpha_{\lambda}^{\prime}(t+2)+\alpha_{\lambda}^{\prime}(t)\right)\right)\right) d t \\
& +2 \sum_{j=1}^{+\infty} \int_{0}^{1} \alpha_{h}^{\prime}(t+j)\left(4(1-\lambda) \alpha_{\lambda}^{\prime}(t+j)\right. \\
& \left.\quad+\lambda\left(2 \alpha_{\lambda}^{\prime}(t+j)+\alpha_{\lambda}^{\prime}(t-2+j)+\alpha_{\lambda}^{\prime}(t+2+j)\right)\right) d t
\end{aligned}
$$

By Lemma 1, for $t \in(0,1)$ the values of $\alpha_{h}^{\prime}(t-1)$ and $\alpha_{h}^{\prime}(t)$ are determined from the initial data, and since they are equal to zero, we have $\alpha_{h}^{\prime}(t-1)=\alpha_{h}(t)=0$. This yields

$$
\begin{aligned}
L_{\lambda}(h)=2 \sum_{k=1}^{+\infty} \int_{0}^{1} \alpha_{h}^{\prime}(t+k)(4(1-\lambda) & \alpha_{\lambda}^{\prime}(t+k) \\
& \left.+\lambda\left(2 \alpha_{\lambda}^{\prime}(t+k)+\alpha_{\lambda}^{\prime}(t-2+k)+\alpha_{\lambda}^{\prime}(t+2+k)\right)\right) d t .
\end{aligned}
$$


If $\lambda>0$ then the roots of the characteristic polynomial $p_{\lambda}$ defined by 32 are $z_{\lambda}$ and $\frac{1}{z_{\lambda}}$. In particular, we have $p_{\lambda}\left(z_{\lambda}\right)=0$. Note that, by Lemma 1, for $t \in(0,1)$ the values of $\alpha_{\lambda}^{\prime}(t-1)$ and of $\alpha_{\lambda}^{\prime}(t)$ are determined from the initial data. By $(24)$, we have

$$
\alpha_{\lambda}^{\prime}(t+1)=-\alpha_{\lambda}^{\prime}(t-1)+u_{\lambda}^{\infty}(t)
$$

for $t \in(0,2)$. Using the representation (7) of $u_{\lambda}^{\infty}(t)$ for $t \in(0,1)$, and using 23), we infer that $\alpha_{\lambda}^{\prime}(t+1)=z_{\lambda} \alpha_{\lambda}^{\prime}(t-1)$ for $t \in(0,1)$. Similarly, using $(24)$, we have $\alpha_{\lambda}^{\prime}(t+2)=-\alpha_{\lambda}^{\prime}(t)+u_{\lambda}^{\infty}(t+1)$ for $t \in(0,1)$. Using the representation (7) of $u_{\lambda}^{\infty}(t)$ for $t \in(1,2)$, and using (23), we infer that $\alpha_{\lambda}^{\prime}(t+2)=z_{\lambda} \alpha_{\lambda}^{\prime}(t)$ for $t \in(0,1)$. It follows that $\alpha_{\lambda}^{\prime}(t+1)=z_{\lambda} \alpha_{\lambda}^{\prime}(t-1)$ for $t \in(0,2)$. By induction, using (34), (8) and (7), we get that

$$
\alpha_{\lambda}^{\prime}(t-1+2 k)=z_{\lambda}^{k} \alpha_{\lambda}^{\prime}(t-1)
$$

for every $t \in(0,2)$ and every $k \in \mathbb{N}$. Therefore, we have obtained that

$$
\lambda \alpha_{\lambda}^{\prime}(t+2+k)+(4-2 \lambda) \alpha_{\lambda}^{\prime}(t+k)+\lambda \alpha_{\lambda}^{\prime}(t-2+k)=p_{\lambda}\left(z_{\lambda}\right) \alpha_{\lambda}^{\prime}(t-2+k)=0,
$$

for every $t \in(0,1)$ and every $k \in \mathbb{N}^{*}$. We conclude that $L_{\lambda}(h)=0$. Concerning the value of the objective functional of $(\mathbf{O C P})_{\lambda}^{\infty}$, we infer that

$$
\begin{aligned}
\int_{0}^{+\infty}\left((1-\lambda)\left(\partial_{x} y(t, 0)\right)^{2}+\lambda u(t)^{2}\right) d t & \\
& \geqslant \int_{0}^{+\infty}\left((1-\lambda)\left(\partial_{x} y_{\lambda}^{\infty}(t, 0)\right)^{2}+\lambda u_{\lambda}^{\infty}(t)^{2}\right) d t,
\end{aligned}
$$

with a strict inequality whenever $h \neq 0$. It follows that $u_{\lambda}^{\infty}$ is the unique optimal solution of $(\mathbf{O C P})_{\lambda}^{\infty}$ for $\lambda>0$. For $\lambda=0$ the result also follows from the representation (33) of $L_{0}(h)$, with the difference that, in this case, the characteristic polynomial is $p_{0}(z)=z$ having the unique root $z_{0}=0$.

The inequality (9) follows from (35), since for the optimal state we have the energy

$$
\begin{aligned}
\int_{0}^{1}\left(\partial_{x} y_{\lambda}^{\infty}(t+2 k, x)\right)^{2}+\left(\partial_{t} y_{\lambda}^{\infty}(t+2 k, x)\right)^{2} d x & =\int_{t+2 k-1}^{t+2 k+1} \alpha_{\lambda}^{\prime}(s)^{2} d s \\
& =\int_{t-1}^{t+1} \alpha_{\lambda}^{\prime}(s+2 k)^{2} d s=\left|z_{\lambda}\right|^{2 k} \int_{t-1}^{t+1} \alpha_{\lambda}^{\prime}(s)^{2} d s
\end{aligned}
$$

for every $t \in(0,2)$ and every $k \in \mathbb{N}$. Theorem 1 is proved for $T=+\infty$.

Remark 7. The computation of the solution with the characteristic polynomial $p_{\lambda}$ is related to techniques used for linear difference equations, or for finite-dimensional linear systems with tridiagonal matrices (see [19]).

\subsection{Proof of Theorem 2}

We assume that $T=2 n$. Using 12 , we have $\frac{1}{2 n}=\frac{1+z_{\lambda}}{2}$, and then, using

$$
u_{1}^{T}(t)= \begin{cases}\frac{1}{2 d(t)}\left(y_{0}^{\prime}(1-t)-y_{1}(1-t)\right), & t \in(0,1), \\ \frac{1}{2 d(t)}\left(y_{0}^{\prime}(t-1)+y_{1}(t-1)\right), & t \in(1,2)\end{cases}
$$


and the representation (7) of $u_{\lambda}^{\infty}$, we infer (13). We have

$$
\left(\int_{0}^{2} u_{\lambda}^{\infty}(t)^{2} d t\right)^{1 / 2} \leqslant\left(1+z_{\lambda}\right)\left(\left\|y_{0}^{\prime}\right\|_{L^{2}(0,1)}+\left\|y_{1}\right\|_{L^{2}(0,1)}\right) .
$$

The inequality (14) follows similarly, using $u_{1}^{T}(t+2 k)=(-1)^{k} u_{1}^{T}(t)$ and [8], since

$$
\left\|u_{1}^{T}-u_{\lambda}^{\infty}\right\|_{L^{2}(2 k, 2 k+2)}=\left.|1-| z_{\lambda}\right|^{k} \mid\left(\int_{0}^{2} u_{\lambda}^{\infty}(t)^{2} d t\right)^{1 / 2} .
$$

The proof for the case $T \notin\{2,4,6, \ldots\}$ works analogously. Theorem 2 is proved.

\section{A general perspective}

In this section we want to illustrate the generality of the turnpike phenomenon in the framework of an evolution equation within the context of semigroup theory. We consider an optimal control problem with an objective function that is again the convex combination of a control cost and a tracking term. In the optimal control problem for the Neumann control of the vibrating string that we have considered in the previous sections the tracking term depends on a boundary observation of the state. In contrast to this situation, the tracking term in Section 4 contains the full state, thus the results that we have presented for the vibrating string cannot be derived easily from the general results given in this section. In contrast to [20], in the optimal control problem that we consider in this section, a terminal constraint appears that prescribes exactly the desired terminal state.

We consider a Hilbert space $\left(X,\langle,\rangle_{X}\right)$ that contains the states and another Hilbert space $\left(U,\langle,\rangle_{U}\right)$ that contains the controls. As in 24] let $A: \mathcal{D}(A) \subset X \rightarrow X$ be the generator of a strongly continuous semigroup $\left(\mathbb{T}_{t}\right)_{t \geqslant 0}$ (for the definitions see also for example [15]) and let $B$ denote an admissible control operator. As stated in [24], $B$ maps $U$ to the space $X_{-1}$, where $X_{-1}$ is defined as the completion of $X$ with respect to the norm $\|z\|_{-1}=\left\|(\beta I-A)^{-1} z\right\|_{X}$ where $\beta \in \rho(A)$ is fixed. In fact the space $X_{-1}$ is independent of the choice of $\beta$. Let an initial state $y_{0} \in X$, and a time $T>0$ be given. As in 24], Proposition 4.2.5 we consider a system that is governed by the differential equation

$$
y^{\prime}(t)=A y(t)+B u(t), t \in[0, T]
$$

with the initial condition $y(0)=y_{0} \in X$. For $u \in L^{2}((0, T) ; U)$ and $t \in[0, T]$, define

$$
\Phi_{t}(u)=\int_{0}^{t} \mathbb{T}_{t-\sigma} B u(\sigma) d \sigma
$$

We assume that there exists a time $T_{\min } \geqslant 0$ such that for all $T>T_{\min }$ the considered system is exactly controllable in the sense that

$$
\operatorname{Ran} \Phi_{T}=X
$$

Let us assume that $T>T_{\min }$, then there exists a control function $u \in L^{2}((0, T) ; U)$ such that the terminal constraint $y(T)=0$ holds. Let $\lambda \in(0,1)$ be given. Consider the problem of optimal exact control

$$
\left\{\begin{array}{l}
\min \int_{0}^{T}\left((1-\lambda)\langle y(t), y(t)\rangle_{X}+\lambda\langle u(t), u(t)\rangle_{U}\right) d t \\
\text { subject to } y^{\prime}(t)=A y(t)+B u(t), t \in[0, T] \\
y(0)=y_{0}, y(T)=0
\end{array}\right.
$$


The static optimal control problem corresponding to 37 is

$$
\left\{\begin{array}{l}
\min \left((1-\lambda)\langle y, y\rangle_{X}+\lambda\langle u, u\rangle_{U}\right) \\
\text { subject to } 0=A y+B u
\end{array}\right.
$$

It is clear that the solution of $(38)$ is zero. The solution of the static optimal control problem (38) determines the turnpike which in our case is $\left(u_{t p}, y_{t p}\right)=(0,0)$.

Let $u_{\lambda}$ denote the optimal control and $y_{\lambda}$ the optimal state for (37). In order to determine the structure of the optimal control we look at the necessary optimality conditions for (37).

Let $u=u_{\lambda}+\delta_{1}$ with a control variation $\delta_{1} \in L^{2}((0, T) ; U)$ that does not change the terminal state. Let $y=y_{\lambda}+\delta_{2}$ denote the corresponding variation of the state, that is we have $\delta_{2} \in$ $C([0, T] ; X)$ with $\delta_{2}(0)=0, \delta_{2}(T)=0$ and $\delta_{2}^{\prime}=A \delta_{2}+B \delta_{1}$. For the objective function in (37) we introduce the notation

$$
J(u, y)=\int_{0}^{T}\left((1-\lambda)\langle y(t), y(t)\rangle_{X}+\lambda\langle u(t), u(t)\rangle_{U}\right) d t .
$$

Then for all $p \in C([0, T] ; X)$ we have $J\left(u_{\lambda}+\delta_{1}, y_{\lambda}+\delta_{2}\right)$

$$
\begin{aligned}
& =J\left(u_{\lambda}, y_{\lambda}\right)+2 \int_{0}^{T} \lambda\left\langle u(s), \delta_{1}(s)\right\rangle_{U}+(1-\lambda)\left\langle y(s), \delta_{2}(s)\right\rangle_{X} d s \\
& +2(1-\lambda) \int_{0}^{T}\left\langle\delta_{2}^{\prime}(s)-A \delta_{2}(s)-B \delta_{1}(s), p(s)\right\rangle_{X} d s \\
& +\int_{0}^{T} \lambda\left\langle\delta_{1}(s), \delta_{1}(s)\right\rangle_{U}+(1-\lambda)\left\langle\delta_{2}(s), \delta_{2}(s)\right\rangle_{X} d s \\
& =J\left(u_{\lambda}, y_{\lambda}\right) \\
& +2 \int_{0}^{T}\left\langle\lambda u(s)-(1-\lambda) B^{*} p, \delta_{1}(s)\right\rangle_{U}+(1-\lambda)\left\langle-p^{\prime}-A^{*} p+y(s), \delta_{2}(s)\right\rangle_{X} d s \\
& +\int_{0}^{T} \lambda\left\langle\delta_{1}(s), \delta_{1}(s)\right\rangle_{U}+(1-\lambda)\left\langle\delta_{2}(s), \delta_{2}(s)\right\rangle_{X} d s .
\end{aligned}
$$

Hence $u_{\lambda}$ can only be an optimal control if there exists a multiplier $p_{\lambda} \in C([0, T] ; X)$ such that the optimality system

$$
\left\{\begin{aligned}
y_{\lambda}^{\prime}= & A y_{\lambda}+B u_{\lambda} \\
p_{\lambda}^{\prime}= & -A^{*} p_{\lambda}+y_{\lambda}, \\
u_{\lambda}= & \frac{1-\lambda}{\lambda} B^{*} p_{\lambda}
\end{aligned}\right.
$$

with the conditions $y_{\lambda}(0)=y_{0}, y_{\lambda}(T)=0$ is satisfied (see also the Lagrange multiplier rule as e.g. in 13 ). By the second equation in the optimality system (39) we get the equation $A y_{\lambda}=A\left(p_{\lambda}^{\prime}+A^{*} p_{\lambda}\right)$. With the third and the first equation in the optimality system (39) this yields $y_{\lambda}^{\prime}-\frac{1-\lambda}{\lambda} B B^{*} p_{\lambda}=y_{\lambda}^{\prime}-B u_{\lambda}=A\left(p_{\lambda}^{\prime}+A^{*} p_{\lambda}\right)$. This yields the second order equation

$$
\left(p_{\lambda}^{\prime}+A^{*} p_{\lambda}\right)^{\prime}=A\left(p_{\lambda}^{\prime}+A^{*} p_{\lambda}\right)+\frac{1-\lambda}{\lambda} B B^{*} p_{\lambda} .
$$

In the remaining discussion we omit the subscript $\lambda$, that is we write $p$ instead of $p_{\lambda}, u$ instead of $u_{\lambda}$ and $y$ instead of $y_{\lambda}$.

\subsection{Skew-adjoint operators}

Let us assume that $A$ is skew-adjoint, i.e., $A^{*}=-A$. In [20, turnpike inequalities for the case of the wave equation where $A^{*}=-A$ are given in Section 4 . Equation 40 yields

$$
p^{\prime \prime}=L p+2 A p^{\prime}
$$


where

$$
L=A A^{*}+\frac{1-\lambda}{\lambda} B B^{*} .
$$

Now we assume that $A$ and $B B^{*}$ are diagonalizable with the same sequence of orthonormal eigenfunctions $\left(\varphi_{k}\right)_{k}$ and that the eigenvalues of $\frac{1-\lambda}{\lambda} B B^{*}$ are bounded from below by $\omega^{2}>0$. Then (41) yields a sequence of ordinary differential equations for $h_{k}(t)=\left\langle p(t), \varphi_{k}\right\rangle_{X}$ namely

$$
h_{k}^{\prime \prime}=\left\langle\varphi_{k}, L \varphi_{k}\right\rangle_{X} h_{k}+2\left\langle\varphi_{k}, A \varphi_{k}\right\rangle_{X} h_{k}^{\prime} .
$$

The characteristic polynomial is

$$
p_{k}(z)=z^{2}-2\left\langle\varphi_{k}, A \varphi_{k}\right\rangle_{X} z-\left\langle\varphi_{k}, L \varphi_{k}\right\rangle_{X}
$$

and since $\left\langle\varphi_{k}, A \varphi_{k}\right\rangle_{X}$ is purely imaginary it has roots $\delta_{k}^{+}, \delta_{k}^{-}$such that $\operatorname{Re}\left(\delta_{k}^{+}\right) \geqslant \omega>0$ and $\operatorname{Re}\left(\delta_{k}^{-}\right) \leqslant-\omega<0$. Thus get the solutions

$$
h_{k}(t)=u_{k} \exp \left(\delta_{k}^{-} t\right)+v_{k} \exp \left(\delta_{k}^{+} t\right) .
$$

The coefficients $u_{k}$ and $v_{k}$ are chosen such that $p^{\prime}(0)=y_{0}+A p(0)$ and $p^{\prime}(T)=A p(T)$, because then we have $y(0)=y_{0}$ and $y(T)=0$. In fact, this yields a constant $C_{\min }$ that is independent of $y_{0}$ and $T$ such that for all $T \geqslant T_{\min }$, we have the inequality

$$
\sum_{k}\left(\left|u_{k}\right|^{2}+\left|\exp \left(2 \delta_{k}^{+} T\right)\right|\left|v_{k}\right|^{2}\right) \leqslant C_{\min }\left\|y_{0}\right\|_{X}^{2}
$$

Using Parseval's equation from the representation

$$
p(t)=\sum_{k} h_{k}(t) \varphi_{k}=\left(\sum_{k} u_{k} \exp \left(\delta_{k}^{-} t\right) \varphi_{k}\right)+\left(\sum_{k} v_{k} \exp \left(\delta_{k}^{+} t\right) \varphi_{k}\right)
$$

we get the inequality

$$
\begin{aligned}
\|p(t)\|_{X} & \leqslant e^{-\omega t}\left(\sum_{k}\left|u_{k}\right|^{2}\right)^{1 / 2}+e^{-\omega(T-t)}\left(\sum_{k}\left|\exp \left(2 \delta_{k}^{+} T\right)\right|\left|v_{k}\right|^{2}\right)^{1 / 2} \\
& \leqslant\left(e^{-\omega t}+e^{-\omega(T-t)}\right) 2 \sqrt{C_{\min }}\left\|y_{0}\right\|_{X} .
\end{aligned}
$$

Inequalities 43)-44 reflect a turnpike inequality for $p$. They state that the norm of $p(t)$ is bounded above by a sum of a part that is exponentially decreasing with time and a second part that

is exponentially increasing towards $T$. Due to $\sqrt{39}$ the optimal control has the form $u=\frac{1-\lambda}{\lambda} B^{*} p$, so it also shows a turnpike structure. Note that the optimal control norms are decreasing with $T$, hence they are uniformly bounded.

\subsection{Self-adjoint operators}

Let us assume that $A$ is self-adjoint. Turnpike inequalities for the parabolic case where $A^{*}=A$ were given in [20, Section 3]. Equation 40, yields

$$
p^{\prime \prime}=L p .
$$

Thus we get

$$
p(t)=\cosh \left(t L^{\frac{1}{2}}\right) p(0)+L^{-\frac{1}{2}} \sinh \left(t L^{\frac{1}{2}}\right) p^{\prime}(0)
$$


with the cosh and sinh operators as defined in [5]. For the optimal state we have

$$
y=p^{\prime}+A^{*} p .
$$

The equations $y(0)=y_{0}, y(T)=0$ yield a system of linear equations for $p(0), p^{\prime}(0)$. In fact we have $p^{\prime}(0)=y_{0}-A^{*} p(0)$ and $p^{\prime}(T)=-A^{*} p(T)$.

Now let us assume that $L$ is diagonalizable and the eigenvalues of $L$ are bounded from below by $\omega^{2}>0$. Then we have the turnpike inequality

$$
\begin{aligned}
\|p(t)\| & \leqslant \frac{1}{2} \exp (-\omega t)\left(\|p(0)\|+\left\|L^{-1 / 2} p^{\prime}(0)\right\|\right) \\
& +\frac{1}{2} \exp (-\omega(T-t))\left(\left\|\exp \left(L^{1 / 2} T\right) p(0)\right\|+\left\|\exp \left(L^{1 / 2} T\right) L^{-1 / 2} p^{\prime}(0)\right\|\right) .
\end{aligned}
$$

The optimal control has the form

$$
u(t)=\frac{1-\lambda}{\lambda} B^{*} \cosh \left(t L^{\frac{1}{2}}\right) p(0)+\frac{1-\lambda}{\lambda} B^{*} L^{-\frac{1}{2}} \sinh \left(t L^{\frac{1}{2}}\right) p^{\prime}(0) .
$$

This means that also the optimal control can be represented as the sum of families of increasing and decreasing exponentials with rates $\omega$. For the optimal state the equation $y=p^{\prime}+A p$ yields a similar representation.

\section{Conclusion}

We have presented optimal Neumann boundary controls for the 1D wave equation for finite an infinite horizons and different weights in the objective function. If the objective function is the control norm and the terminal state is prescribed exactly, the control action is distributed periodically over the whole time horizon and coincides with the control given by the Hilbert Uniqueness Method. In contrast, if the objective function involves an additional tracking term the optimal control action is essentially concentrated at the starting time 0 and at the terminal time $T$, and in-between it is exponentially close to 0 . The control is the sum of an exponentially decreasing term and of an exponentially increasing one. If the time horizon is infinite only the first term remains, and the optimal control exponentially stabilizes the system, according to the classical Riccati theory. The norms of the control action and of the optimal state decay then exponentially in time. These results show that as soon as the objective functional of the optimal control problems for the considered system contains a nontrivial tracking term, the optimal solution has a special behavior referred to as the turnpike phenomenon. We have shown that, if the final time $T$ is a positive even integer, there exists a weight $\lambda$ such that the solution of the problem of exact control with minimal control norm coincides with the solution of the infinite horizon optimal control problem along the time interval $[0,2]$. This result justifies a receding horizon control strategy, where the first part of a finite horizon optimal control is used and then the procedure is updated in order to control the system over an infinite time horizon.

As already said, the turnpike property has been much investigated in finite dimension (see 23 and references therein for a general result). In the infinite-dimensional setting, in 20 distributed control has been considered both for the heat equation and the wave equation. The turnpike phenomenon put in evidence in the present paper shows an interesting qualitative bifurcation of the HUM control as soon as the objective functional involves a tracking term.

Several open questions are in order. First of all, it makes sense to consider an objective functional in which the tracking term is replaced with a discrepancy between the solution and a time-independent function, which is not necessarily a steady-state. According to the results of [23], 
we expect then that the turnpike property still holds true, and that, in large time, the optimal trajectory remains essentially close to the optimal steady-state, defined as the closest steady-state to the objective. However, in this case, it might be difficult to derive explicit formulas as done in the present paper. Moreover, here we only considered a functional penalizing the normal derivative at $x=0$, and then we can only consider a time-independent function that is a steady-state, as said in Remark 4. But if instead, we were considering for instance the full norm in $H^{1}(0,1)$, then we could consider in the objective functional a term of the form $\|y(t, \cdot)-a(\cdot)\|^{2}$, where $a(\cdot)$ need not be a steady-state. Then, what can be expected is that, in large time, the optimal trajectory remains essentially close to the steady-state of the form $\sigma x$ that is the closest possible to the target $a(\cdot)$.

For more general multi-D wave equations, the situation is open. Even if explicit computations can only be done in specific cases, we expect that the turnpike phenomenon is generic within the class of optimal control problems for controllable wave equations, and that HUM controls characterized by the adjoint system develop a quasi-periodic pattern, but when characterized by a more robust optimality cost, then, satisfy the turnpike property.

Another open issue is the investigation of semilinear wave equations (see [2]), for which steadystates may play an important role. Of course, in that case, we cannot expect that the turnpike property hold globally, but it should also hold as well at least in some neighborhood of an optimal steady-state (see discussions in [23]).

Acknowledgment. We thank the referees for their comments that helped to improve the paper. This work is supported by DFG in the framework of the Collaborative Research Centre CRC/Transregio 154, Mathematical Modelling, Simulation and Optimization Using the Example of Gas Networks, project $\mathrm{C} 03$ and project A03. The third author was funded by Grants FA9550-14-1-0214 of the EOARD-AFOSR, FA9550-15-1-0027 of AFOSR and MTM2014-52347 of the MINECO (Spain) and a Humboldt Award at the University of Erlangen-Nurenberg.

\section{References}

[1] D. A. Carlson, A. Haurie and A. Jabrane, Existence of overtaking solutions to infinite dimensional control problems on unbounded time intervals, SIAM J. Control Optim. 25, 1517$1541,1987$.

[2] J.-M. Coron, E. Trélat, Global steady-state stabilization and controllability of $1 D$ semilinear wave equations, Comm. Cont. Math. 8, pp. 535-567, 2006.

[3] T. Damm, L. Grüne, M. Stieler and K. Worthmann, An exponential turnpike theorem for dissipative discrete time optimal control problems, SIAM J. Control Optim. 52 (2014), no. 3, 1935-1957.

[4] R. Dorfman, P.A. Samuelson, R. M. Solow, Linear Programming and Economic Analysis, New York: McGraw-Hill, 1958.

[5] H.O Fattorini, Ordinary differential equations in linear topological spaces, Pts. II, J. Differ. Equations, 6, pp. 50-70, 1969.

[6] F. Flandoli, I. Lasiecka, R. Triggiani, Algebraic Riccati equations with non-smoothing observation arising in hyperbolic and Euler-Bernoulli boundary control problems, Annali di Matematica Pura ed Applicata 153, pp. 307-382, 1988. 
[7] M. Gugat, Norm-Minimal Neumann Boundary Control of the Wave Equation, Arabian Journal of Mathematics, 4, 41-58, 2015.

[8] M. Gugat, Optimal Boundary Control and Boundary Stabilization of Hyperbolic Systems, SpringerBriefs in Control, Automation and Robotics, Birkhäuser, Basel, 2015.

[9] M. Gugat, G. Leugering, G. Sklyar, $L^{p}$-optimal boundary control for the wave equation, SIAM J. Control Optim. 44, pp. 49-74, 2005.

[10] M. Gugat, M. Tucsnak, An example for the switching delay feedback stabilization of an infinite dimensional system: The boundary stabilization of a string, Syst. Cont. Lett. 60, pp. 226-233, 2011.

[11] M. Gugat, Optimal boundary feedback stabilization of a string with moving boundary, IMA Journal of Mathematical Control and Information 25 (2008), 111-121.

[12] A. Haurie, Optimal control on an infinite time horizon. The turnpike approach, J. Math. Economics 3 (1976), 81-102.

[13] I. Kazufumi and K. Kunisch, Multiplier approach to variational problems and applications., Advances in Design and Control, 15. Society for Industrial and Applied Mathematics (SIAM), Philadelphia, PA, 2008.

[14] W. Krabs, On time-minimal distributed control of vibrations, Appl. Math. Optim. 19, pp. 65-73, 1989.

[15] R. Triggiani, I. Lasiecka, Control Theory for Partial Differential Equations: Volume 2, Abstract Hyperbolic-like Systems over a Finite Time Horizon, Cambridge University Press, Cambridge, UK, 2001

[16] J.-L. Lions, Exact controllability, stabilization and perturbations for distributed systems, SIAM Rev. 30, pp. 1-68, 1988.

[17] M. Mammadov, R.J. Evans, Turnpike theorem for terminal functionals in infinite horizon optimal control problems, Journal of Mathematical Analysis and Applications, 428, no. 2, $1147-1160$.

[18] L.W. McKenzie, Turnpike Theory, Econometrica 44 (1976), 841-865.

[19] G. Meurant, A Review on the Inverse of Symmetric Tridiagonal and Block Tridiagonal Matrices SIAM. J. Matrix Anal. Appl. 13(3), pp. 707-728, 1992.

[20] A. Porretta and E. Zuazua, Long Time versus Steady State Optimal Control, SIAM J. Control and Optimization 51, 4242-4273, 2013.

[21] A. Rapaport, P. Cartigny, Turnpike theorems by a value function approach, ESAIM: Control Optim. Calc. Var. 10 (2004), 123-141.

[22] D.L. Russell, Nonharmonic Fourier series in the control theory of distributed parameter systems, J. Math. Anal. Appl. 18, pp. 542-560, 1967.

[23] E. Trélat, E. Zuazua, The turnpike property in finite-dimensional nonlinear optimal control, J. Differential Equations 258, 81-114, 2015.

[24] M. Tucsnak and G. Weiss, Observation and Control for Operator Semigroups, Birkhäuser Advanced Texts, Basel, Switzerland, 2009. 
[25] A. Zaslavski, Turnpike properties in the calculus of variations and optimal control, Non-convex Optimization and its Applications, vol. 80, Springer, New York, 2006.

[26] A. Zaslavski, Structure of approximate solutions of optimal control problems, Springer Briefs in Optimization. Springer, New York, 2013. viii+127 pp.

[27] A. Zaslavski, Turnpike phenomenon and infinite horizon optimal control, Springer Optimization and Its Applications, 99. Springer, Cham, 2014. $\mathrm{x}+370 \mathrm{pp}$.

[28] A. Zaslavski, Turnpike theory of continuous-time linear optimal control problems, Springer Optimization and Its Applications, 104. Springer, Cham, 2015. ix+296 pp.

[29] E. Zuazua, Propagation, observation, and control of waves approximated by finite difference methods, SIAM Review 47, pp. 197-243, 2005. 